\title{
La thrombectomie : la deuxième révolution dans le traitement de l'infarctus cérébral
}

\author{
Thrombectomy: The Second Revolution in the Treatment of Ischemic Stroke
}

\author{
M. Mazighi \\ Reçu le 28 mai 2015 ; accepté le 24 juillet 2015 \\ (c) SRLF et Lavoisier SAS 2015
}

Mille neuf cent quatre-vingt-quinze a été l'année de la thrombolyse intraveineuse (IV), 2015 est celle de la thrombectomie mécanique (TM). Dans l'étude National Institute of Neurological Disorders and Stroke t-PA Study [1], la thrombolyse IV a montré son efficacité, à la phase aiguë de l'infarctus cérébral, en augmentant de $15 \%$ la probabilité d'avoir un pronostic favorable par rapport au placebo. Vingt ans plus tard, cinq études randomisées montrent le bénéfice de la TM (capture mécanique du caillot par voie endovasculaire) associée à la thrombolyse IV par rapport à la prise en charge médicale usuelle (incluant la thrombolyse IV seule).

Multicenter Randomized Clinical trial of Endovascular Treatment in the Netherlands (MR CLEAN) est la première étude positive publiée dans le New England Journal of Medicine en décembre 2014 [2]. À la suite de cette « bombe », des analyses intermédiaires ont été à l'origine de l'arrêt de trois études : Endovascular treatment for Small Core and Anterior circulation Proximal occlusion with Emphasis on minimizing CT to recanalization times (ESCAPE) [2], Extending the time for Thrombolysis in Emergency Neurological Deficits with Intra-arterial therapy (EXTEND-IA) [3] et Solitaire With the Intention For Thrombectomy as PRIMary Endovascular Treatment (SWIFT-PRIME) [4]. La dernière étude, Endovascular Revascularization With Solitaire Device Versus Best Medical Therapy in Anterior Circulation Stroke Within 8 Hours (REVASCAT) [5], a été arrêtée pour perte d' " équipoise » devant les résultats positifs des études précédentes. Dans ces cinq études, le nombre de patients inclus était faible (500 patients pour MR CLEAN [2], 70 pour EXTEND-IA [3], 316 pour ESCAPE [4], 196 dans SWIFT-PRIME [5] et 206 pour REVASCAT [6]), en partie parce que la majorité

\footnotetext{
M. Mazighi $(\bowtie)$

Service de neurologie, hôpital Lariboisière,

2, rue Ambroise Paré, 75010 Paris

e-mail : mikael.mazighi@aphp.fr
}

Pôle neurosensoriel, INSERM 1148, université Paris 7 Denis Diderot-Sorbonne Paris Cité d'entre elles n'ont pas été menées jusqu'à leur terme. Parmi les éléments importants de ces études, il est à noter que la TM a été réalisée dans 73 à $100 \%$ des cas après administration de la thrombolyse IV [2-6]. Il n'y avait pas de limite d'âge, sauf pour deux études (SWIFT-PRIME et REVASCAT), qui ont imposé une barre à 80 ans. Les patients inclus avaient un déficit neurologique important avec un score NIHSS (score neurologique de gravité initiale et pronostique) médian de 17. Même si les fenêtres thérapeutiques variaient de 6 à 12 heures, plus de $80 \%$ des patients traités par TM l'ont été dans les six heures suivant le début des symptômes. Sur le plan technique, les dispositifs de TM de référence, les stentrievers (stents déployés mais non implantés, qui plaquent le caillot contre la paroi de l'artère, rétablissent un flux artériel et permettent de capturer le caillot lors du retrait), ont été utilisés dans la majorité des cas (81 à $100 \%$ ) [1-5]. La présence d'une occlusion artérielle proximale de la circulation antérieure (artère carotide interne, l'artère cérébrale moyenne) avait été confirmée sur une imagerie non invasive. Il s'agit là de points clés, car dans les études négatives publiées également dans le New England Journal of Medicine en 2013 [7-9], les patients n'avaient pas été systématiquement sélectionnés sur la présence d'une occlusion artérielle intracrânienne et les stentrievers avaient été utilisés de façon marginale.

Dans ces cinq études, la TM a été associée à une évolution favorable (évaluée par l'échelle modifiée de Rankin : 0-2 à 3 mois) dans $33 \%$ des cas pour MR CLEAN, $71 \%$ pour EXTEND-IA, $53 \%$ pour ESCAPE, $60 \%$ pour SWIFTPRIME et $44 \%$ pour REVASCAT. Par comparaison, elle a été dans le bras contrôle de $19 \%$ pour MR CLEAN, $40 \%$ pour EXTEND-IA, $29 \%$ pour ESCAPE, et $28 \%$ pour REVASCAT. Pour obtenir un bénéfice de la TM, il fallait traiter huit patients dans MR CLEAN, trois dans EXTENDIA, quatre dans ESCAPE, quatre pour SWIFT-PRIME et 6,5 dans REVASCAT. Dans une seule étude, (ESCAPE), un effet sur la mortalité a été observé, avec une réduction relative du risque de près de $50 \%$ avec la TM $(10,4 \%$ vs $19 \%, \mathrm{p}=0,04)$. Il convient de préciser ici que cet effet sur le pronostic est 
obtenu pour des temps médians de reperfusion inférieurs à 6 heures. Il faut donc s'interroger sur la reproductibilité de ces résultats au-delà des 6 heures. Par ailleurs, plusieurs questions restent en suspens, notamment quant à la limite d'âge. Les analyses post hoc montrent également un bénéfice pour les patients de plus de 80 ans, mais ceux-ci étaient minoritaires. Il existe aussi un débat sur la place de l'anesthésie générale. Par exemple, dans MR CLEAN, les patients traités par TM ont montré une évolution à trois mois plus défavorable en cas d'anesthésie générale. Plusieurs études en cours ou à venir vont tenter de clarifier l'impact du type d'anesthésie en cas de $\mathrm{TM}$, en éliminant certains biais liés à la gravité de l'infarctus cérébral initial ou à l'absence du maintien de la pression de perfusion cérébrale lors de l'induction.

La TM avec les stentrievers associée à la thrombolyse IV est désormais le traitement de référence de l'infarctus cérébral avec occlusion proximale de la circulation cérébrale antérieure [10]. Il s'agit là d'un changement de paradigme dans le traitement de l'infarctus cérébral. Le défi organisationnel qui s'ouvre à nous est immense, pour offrir ce type de thérapeutique au plus grand nombre de patients. L'équation peut se résumer par une prise en charge multidisciplinaire (neurologues, radiologues, anesthésistes, réanimateurs, urgentistes, SAMU), faisant appel à des ressources rares, dont l'accessibilité doit être mise en œuvre dans des délais extrêmement courts.

Liens d'intérêts : L'auteur déclare un lien d'intérêt avec la société Covidien : prise en charge des frais pour la participation à des congrès, présentation orale.

\section{Références}

1. 1995) Tissue plasminogen activator for acute ischemic stroke. The National Institute of Neurological Disorders and Stroke Study Group. N Engl J Med 333:1581-7

2. Berkhemer OA, Fransen PS, Beumer D, et al (2015) A randomized trial of intraarterial treatment for acute ischemic stroke. N Engl J Med 372:11-20

3. Campbell BC, Mitchell PJ, Kleinig TJ, et al (2015) Endovascular Therapy for Ischemic Stroke with Perfusion-Imaging Selection. N Engl J Med 372:1009-18

4. Goyal M, Demchuk AM, Menon BK, et al (2015) Randomized Assessment of Rapid Endovascular Treatment of Ischemic Stroke. N Engl J Med 372:1019-30

5. Saver JL, Goyal M, Bonafe A, et al (2015) Stent-retriever thrombectomy after intravenous t-PA vs. t-PA alone in stroke. $\mathrm{N}$ Engl $\mathrm{J}$ Med 372:1019-30

6. Jovin TG, Chamorro A, Cobo E, et al (2015) REVASCAT Trial Investigators.Thrombectomy within 8 Hours after Symptom Onset in Ischemic Stroke. N Engl J Med 372:2296-306

7. Ciccone A, Valvassori L, Nichelatti M, et al (2013) Endovascular treatment for acute ischemic stroke. N Engl J Med 368:904-13

8. Broderick JP, Palesch YY, Demchuk AM, et al (2013) Endovascular therapy after intravenous t-PA versus t-PA alone for stroke. N Engl J Med 368:893-903

9. Kidwell CS, Jahan R, Gornbein J, et al (2013) A trial of imaging selection and endovascular treatment for ischemic stroke. N Engl J Med 368:914-23

10. Consensus statement on mechanical thrombectomy in acute ischemic stroke - ESO-Karolinska Stroke Update 2014 in collaboration with ESMINT and ESNR. http://2014.strokeupdate.org/ consensus-statement-mechanical-thrombectomy-acute-ischemicstroke 